\title{
The Efficacy of Alendronate Treatment in Ambulatory Stroke Patients
}

Nihal ÖZARAS', Saliha EROĞLU DEMIR'1, Aysun ARSLAN²

${ }^{1}$ Department of Physical Medicine and Rehabilitation, Bezmialem Vakif University School of Medicine, İstanbul, Turkey

${ }^{2}$ Clinic of Neurology, Dr. Lütfi Kurdar Kartal Training and Research Hospital, İstanbul, Turkey

\section{ABSTRACT}

Objective: Hip fracture is a serious complication that occurs after stroke. The incidence of hip fracture in stroke patients is $2-4$ times higher than that in normal people and most of these fractures occur on the paretic side. A significant bone loss on the paretic side of stroke patients has been reported. The aim of this study was to evaluate the efficacy of alendronate therapy in ambulatory stroke patients.

Methods: Ambulatory stroke patients who have been followed in the outpatient clinic $(n=20)$ were enrolled in the study. Their bone mineral density (BMD) values and laboratory parameters were retrospectively evaluated according to the medications given for osteoporosis. The analysis was performed by comparing the results of patients who had been given (Group 1) and not given (Group 2) bisphosphonate (alendronate) for a one-year period.

Results: Measurements performed after a one year interval revealed that the BMD values of lumbar region and paretic and nonparetic femur neck were increased in Group 1, whereas these values were decreased in Group 2. However, when the drug use was considered, the changes were statistically significant only for the lumbar region $(\mathrm{p}=0.004)$.

Conclusion: Alendronate treatment may have a beneficial effect on the hemiosteoporosis of ambulatory stroke patients.

Keywords: Osteoporosis, bisphosphonate, alendronate, stroke, ambulatory

\section{Introduction}

Hip fracture is a serious and common complication after cerebrovascular stroke (1). The incidence of hip fracture in stroke patients is $2-4$ times higher than that in an age-matched reference population, and most of these fractures occur on the paretic side $(2,3)$.

Because of advances in the diagnosis and treatment of stroke, the number of disabled stroke survivors has increased. Prevention of hip fracture is important in the management of these patients. Osteoporosis and increased falls due to the motor impairments are the risk factors for hip fracture (1-4). During the first year after stroke, increased bone resorption was determined (5). A significant bone loss (3\%) on the paretic side of patients who would be able to walk within the first week of stroke has been reported (6). Therefore, a regular follow-up for osteoporosis must be considered in these patients and the necessary treatment must be given immediately $(1,3,7)$. Bisphosphonates and vitamin $\mathrm{D}$ preparations are the first-line treatment for patients with high fracture risk (8). The aim of this study was to evaluate the efficacy of osteoporosis therapy, particularly using bisphosphonates, in ambulatory stroke patients.

\section{Methods}

Subjects: The subjects were chosen from patients who had been followed up from our stroke unit according to their stroke duration (within the first year), age ( $>60$ years), and ambulation status (could walk with or without aids). The exclusion criteria were history of previous strokes, renal or hepatic insufficiency, cardiac failure, previous osteoporotic fracture, unilateral bone disease asymmetrically affecting bone mineral density (BMD), and ongoing treatment with drugs known to alter bone metabolism. 
Methods: In our stroke unit, the patients were assessed for stroke risk factors (smoking, alcohol use, medications, body mass index, etc.) and evaluated using the Barthel Index (BI) and Scandinavian Stroke Scale (SSS) for the determination of functional status and clinical severity of hemiplegia to determine realistic goals for rehabilitation. The awareness of increased hip fracture risk in stroke patients made us evaluate osteoporosis in these patients.

Bone mineral density of lumbar spine and bilateral femoral necks were assessed using dual-energy X-ray absorptiometry (DXA, Lunar-DPX) and laboratory parameters related to osteoporosis [serum osteocalcin, alkaline phosphatase (ALP), calcium, and parathyroid hormone (PTH) levels and urinary deoxypyridinoline (urine DPD) levels] were analyzed routinely. If the patient had a T-score of $\leq(-2.5)$ at the femoral neck, total hip, or lumbar spine, bisphosphonate treatment (usually alendronate) is given, apart from vitamin $\mathrm{D}$ and calcium supplementation, as recommended by National Osteoporosis Foundation (9).

Only 20 of the 150 evaluated stroke patients were included in the study based on exclusion and inclusion criteria. The mean duration of the stroke was $3.70 \pm 2.16$ months, and there were no significant differences between the paretic and nonparetic femur neck BMDs at the beginning of the follow-up. The subjects who used and did not use alendronate were classified as Group 1 and 2, respectively. Both Group 1 and 2 received 1 $\mathrm{mcg}$ of alfacalcidol and $500 \mathrm{mg}$ ionized calcium supplementation daily.

In this study, the results of yearly evaluation of the patients were retrospectively analyzed with respect to osteoporosis. The values were classified into two categories according to the administration of alendronate and were statistically compared.

\section{Statistical analysis}

The data were analyzed using the Statistical Package for the Social Sciences (SPSS Inc., Version 16.0, Chicago, USA). Student's $t$-test or Mann-Whitney U test was used for continuous variables, and general linear model test with repeated measures was used for the analysis of the follow-up data of BMD values and osteocalcin, deoxypyridinoline, PTH, ALP, and calcium levels in each group of treatment.

\section{Results}

There were no statistically significant differences between Group 1 and 2 with respect to age, duration of stroke, BI, and SSS. BMD values with a one-year interval were given in Table 1. At the end of the one year, BMD values of lumbar region and paretic and nonparetic femur neck were increased

Table 1. Bone mineral density changes in the study groups

\begin{tabular}{|c|c|c|c|c|c|c|}
\hline & \multicolumn{3}{|c|}{ Group $1(n=7)$} & \multicolumn{3}{|c|}{ Group $2(n=13)$} \\
\hline & BMD-1 & BMD-2 & $\%$ change, p & BMD-1 & BMD-2 & $\%$ change, $p$ \\
\hline Paretic femur neck & $0.733 \pm 0.08$ & $0.765 \pm 0.07$ & $+4.3 \%,>0.05$ & $0.878 \pm 0.11$ & $0.842 \pm 0.11$ & $-4.1 \%,>0.05$ \\
\hline Normal femur neck & $0.740 \pm 0.09$ & $0.773 \pm 0.09$ & $+4.4 \%,>0.05$ & $0.865 \pm 0.13$ & $0.852 \pm 0.11$ & $-1.5 \%,>0.05$ \\
\hline Lumbar spine & $0.897 \pm 0.05$ & $0.972 \pm 0.05$ & $+8.4 \%,=0.004$ & $1.168 \pm 0.18$ & $1.142 \pm 0.16$ & $-2.2 \%,>0.05$ \\
\hline \multicolumn{7}{|c|}{ BMD: Bone mineral density } \\
\hline \multicolumn{7}{|c|}{ BMD-1 is the BMD value at the beginning of the year } \\
\hline \multicolumn{7}{|c|}{ BMD- 2 is the BMD value at the end of the year } \\
\hline
\end{tabular}

Table 2. Serum alkaline phosphatase, calcium, parathyroid hormone, osteocalcin, and urine deoxypyridinoline level changes in 12 months of follow-up

\begin{tabular}{|c|c|c|c|c|c|c|}
\hline & \multicolumn{3}{|c|}{ Group $1(n=7)$} & \multicolumn{3}{|c|}{ Group $2(n=13)$} \\
\hline & Normal values & $1^{\text {st }}$ value & $2^{\text {nd }}$ value & $1^{\text {st }}$ value & $2^{\text {nd }}$ value & $\mathrm{p}^{*}$ \\
\hline PTH (pg/mL) & $7-53$ & $42.2 \pm 24$ & $52.2 \pm 25$ & $24.5 \pm 13$ & $69.8 \pm 41$ & 0.039 \\
\hline Osteocalcin (ng/mL) & $3.1-13.7$ & $10.2 \pm 6.8$ & $3.7 \pm 2.5$ & $7.7 \pm 4.4$ & $10.5 \pm 5.9$ & 0.003 \\
\hline DPD (DPD/mM creatinine) & $2.3-7.4$ & $10.73 \pm 4.5$ & $8.84 \pm 2.4$ & $12.05 \pm 5.4$ & $8.13 \pm 1.9$ & $>0.05$ \\
\hline Calcium (mg/dL) & $8.6-10.2$ & $9.1 \pm 0.8$ & $9.5 \pm 0.2$ & $9.0 \pm 0.7$ & $9.8 \pm 0.5$ & $>0.05$ \\
\hline $\operatorname{ALP}(U / L)$ & $55-298$ & $129 \pm 76$ & $191 \pm 31$ & $108 \pm 35$ & $240 \pm 35$ & 0.016 \\
\hline $\begin{array}{l}1^{\text {st }} \text { value is the value measured } \\
2^{\text {nd }} \text { value is the value measurec } \\
\text { *p value represents the chang } \\
\text { Repeated Measures) } \\
\text { ALP: Serum alkaline phosphat }\end{array}$ & $\begin{array}{l}t \text { the beginning of th } \\
t \text { the end of the year } \\
\text { in serum and urine b } \\
\text {; PTH: parathyroid } h\end{array}$ & $\begin{array}{l}\text { emical indice } \\
\text { one; DPD: de }\end{array}$ & $\begin{array}{l}\text { en the grou } \\
\text { dinoline }\end{array}$ & considering th & Jse (by Gener & Model Test \\
\hline
\end{tabular}


in the Group 1, whereas these values were decreased in Group 2. These increases in BMD values were statistically significant only for the lumbar region $(\mathrm{p}=0.004)$. The $\mathrm{BMD}$ values measured at the end of the year were lower in the paretic femur neck than in the normal femur neck in both groups; however, these differences were not statistically significant $(p>0.05)$ (Table 1).

Serum calcium, ALP, PTH, osteocalcin, and urine DPD level changes are presented in Table 2. Serum calcium and ALP levels were within normal ranges in both groups. Serum PTH levels increased in both Group 1 and 2, however it was increased above the normal ranges in Group 2. Serum osteocalcin levels increased in Group 2 and decreased in Group 1, but remained within the normal ranges in both groups. Urine DPD levels decreased in both groups, and the decreased values were still higher than the normal limits. When the drug use was considered, the changes in serum PTH, osteocalcin, and ALP levels between the groups were statistically significant.

During the analyzed year, none of the patients had osteoporotic fracture.

\section{Discussion}

Several studies have reported a reduction in BMD on the affected side and hemiosteoporosis in hemiplegic stroke patients $(1,10,11)$. Immobilization was determined as a major factor in the development of hemiosteoporosis; however, some other factors such as sympathetic nervous activity that affects the paretic and nonparetic side unequally may be of importance. The result of a study reporting a significant bone loss (3\%) on the paretic side of patients who would be able to walk within the first week after stroke was supporting this approach (6). In the study by Ramnemark et al. (1), a BMD reduction of both lower extremities, which was more pronounced on the paretic side, was found after a 12-month follow-up of patients with severe hemiparesis. These patients were generally immobile, and BMD reduction was attributed to immobilization. Another study by Jorgensen et al. (6) demonstrated a significant BMD reduction only on the paretic side of stroke patients who were able to walk, and it was suggested that the amount of weight borne on the paretic leg was the determinant for bone loss. In our study, all the patients were ambulatory and there were no significant differences between the paretic and nonparetic femur neck BMDs at the beginning of the follow-up. At the end of the one year, both paretic and nonparetic femur neck BMD values increased in Group 1 and decreased in Group 2, and in both the groups, the paretic femur neck BMD values were lower than the nonparetic femur neck BMD values; however, these differences were statistically insignificant in both groups. The BMD reduction in nonparetic femur neck of Group 2 in our study was not unexpected because a mean decrease in BMD of $1 \%-15 \%$ per year was reported in healthy elderly people (6).
At the start of the follow-up, serum PTH and osteocalcin levels were within the normal ranges in Group 1 and 2. In stroke patients, immobilization-induced hypercalcemia may be seen, and it may inhibit PTH level. Depressed osteocalcin levels may be observed in these patients, which could be explained by immobilization itself (12). Our patients were not immobile, and the duration of the disease was short. Possibly for these reasons, both PTH and osteocalcin levels were normal at the beginning. Urine DPD levels of the patients were higher than the normal ranges. Increased bone resorption was reported during the first year after hemiplegic stroke (6); this seems to be also present in ambulatory stroke patients.

Immobility-induced hypercalcemia correlated with BI was reported in stroke patients (10). In our study, all the patients were mobilized, and possibly for this reason, the serum calcium levels of both the groups were in normal range at the beginning and end of the year.

At the end of the one-year follow-up, in Group 2, serum ALP and PTH levels significantly increased. These increases may reflect continuing high bone turnover in Group 2. When the drug use was considered, the changes in serum PTH, osteocalcin, and ALP levels between the groups were statistically significant and may reflect the beneficial effect of alendronate on the bone metabolism. The urine DPD levels were still higher than normal in both groups; however, because the bone resorption also continues in healthy elderly people, these levels were not contradictory (6).

The bisphosphonates inhibit osteoclast-mediated bone resorption (3). The probable beneficial effects of the bisphosphonates were suggested in the prevention of hemiosteoporosis in stroke patients $(1,11)$. In a study, after etidronate administration, prevention of BMD decrease in the paretic femoral neck of stroke patients with low activity of daily living (ADL) was reported, whereas no beneficial effect was seen in patients with high ADL (11). Another study investigating the effectiveness of risedronate in hemiplegic patients revealed that it increases BMD and decreases the occurrence of hip fracture in this population (13). Similar to our research, there is a study comparing the effects of alendronate and alphacalcidiol in stroke patients; however, the subjects in that study were in the chronic stage of the disease (mean duration was $>3$ years) and the BMD measurements were done by computed X-ray densitometry. In this study, over 12 months, BMD in the alendronate group considerably increased, whereas it was decreased in the other group, and the difference in the percent change in BMD between the two groups was found to be significant $(p<0.001)$ (14). In our study, we had a chance to evaluate the effect of these medications at the very early stage of hemiplegia (mean duration was 3.70 \pm 2.16 months), and we found that the BMD values of both paretic and nonparetic femur neck and lumbar spine were increased over the 12 months in Group 1, whereas these values were decreased in Group 2. Possibly because of the low number of patients, these changes were statistically significant only in the lumbar region. 
Because osteoporosis is an important risk factor for hip fracture (1-4), alendronate is believed to prevent fracture by preventing osteoporosis. However, interestingly, a Cochrane review reports that alendronate also reduce the rate of falls and numbers of fallers (15). This effect may contribute to the reduction of hip fractures.

\section{Conclusion}

We enrolled stroke patients with ability to walk in the study, and we detected the possible beneficial effect of alendronate for hemiosteoporosis. This effect may be seen more noticeably by an investigation with a high number of patients.

Ethics Committee Approval: Since the study includes retrospective datae review, an ethical committee approval is not needed.

Informed Consent: Since the study includes retrospective datae review, informed consent is not needed.

Peer-review: Externally peer-reviewed.

Author Contributions: Concept - N.Ö.; Design - N.Ö., S.E.D., A.A.; Supervision - N.Ö.; Funding - N.Ö., S.E.D.; Materials - S.E.D., A.A.; Data Collection and/or Processing - N.Ö.; Analysis and/or Interpretation - N.Ö., S.E.D., A.A.; Literature Review - N.Ö, S.E.D., A.A.; Writer - N.Ö., S.E.D., A.A.; Critical Review - N.Ö., S.E.D., A.A

Conflict of Interest: No conflict of interest was declared by the authors.

Financial Disclosure: The authors declared that this study has received no financial support.

\section{References}

1. Ramnemark A, Nyberg L, Lorentzon R, Englund U, Gustafson Y. Progressive hemiosteoporosis on the paretic side and increased bone mineral density in nonparetic arm the first year after severe stroke. Osteoporos Int 1999; 9: 269-75. [CrossRef]
2. Ramnemark A, Nyberg L, Borssen B, Olsson T, Gustafson Y. Fractures after stroke. Osteoporos Int 1998; 8: 92-5. [CrossRef]

3. Poole KES, Reeve J, Warburton EA. Falls, fractures, and osteoporosis after stroke, time to think about protection. Stroke 2002; 33: 1432-6. [CrossRef]

4. Aizen E. Falls in patients with stroke. Harefuah 2014; 153: 195-8.

5. Liu M, Tsuji T, Higuchi Y, Domen K, Tsujiuchi K, Chino N. Osteoporosis in hemiplegic stroke patients as studied with dual-energy X-ray absorptiometry. Arch Phys Med Rehabil 1999; 80: 1219-26. [CrossRef]

6. Jørgensen L, Jacobsen BK, Wilsgaard T, Magnus JH. Walking after stroke: does it matter? Changes in bone mineral density within the first 12 months after stroke. A longitudinal study. Osteoporos Int 2000; 11: 381-7. [CrossRef]

7. Sato Y, Maruoka H, Oizumi K, Kikuyama M. Vitamin D deficiency and osteopenia in the hemiplegic limbs of stroke patients. Stroke 1996; 27: 2183-7. [CrossRef]

8. Brown JP, Morin S, Leslie W, Papaioannou A, Cheung AM, Davison KS, et al. Bisphosphonates for treatment of osteoporosis: expected benefits, potential harms, and drug holidays. Can Fam Physician 2014; 60: 324-33.

9. National Osteoporosis Foundation. Clinician's Guide to Prevention and Treatment of Osteoporosis. Washington, DC: National Osteoporosis Foundation; 2014.

10. Sato Y, Fujimatsu Y, Kikuyama M, Kaji M, Oizumi K. Influence of immobilization on bone mass and bone metabolism in hemiplegic elderly patients with a long-standing stroke. J Neurol Sci 1998; 156: 205-10. [CrossRef]

11. Ikai T, Uematsu M, Eun SS, Kimura C, Hasegawa C, Miyano S. Prevention of secondary osteoporosis postmenapouse in hemiplegia. Am J Phys Med Rehabil 2001; 80: 169-74. [CrossRef]

12. Yavuzer G, Ataman S, Suldur N, Atay M. Bone mineral density in patients with stroke. Int J Rehabil Res 2002; 25: 235-9. [CrossRef]

13. Sato Y, Iwamoto J, Kanoko T, Satoh K. Risedronate sodium therapy for prevention of hip fracture in men 65 years or older after stroke. Arch Intern Med 2005; 165: 1743-8. [CrossRef]

14. Sato Y, Iwamoto J, Honda Y. An open-label trial comparing alendronate and alphacalcidol in reducing falls and hip fractures in disabled stroke patients. J Stroke Cerebrovasc Dis 2011; 20: 41-6. [CrossRef]

15. Verheyden GS, Weerdesteyn V, Pickering RM, Kunkel D, Lennon $S$, Geurts AC, et al. Interventions for preventing falls in people after stroke. Cochrane Database Syst Rev 2013; 5: CD008728. [CrossRef] 